\title{
Explicit Asymmetric Bounds for Robust Stability of Continuous and Discrete-Time-Systems
}

\author{
Zhiqiang Gao \\ Cleveland State University, Z.GAO@csuohio.edu \\ P J. Antsaklis \\ Cleveland State University
}

Follow this and additional works at: https://engagedscholarship.csuohio.edu/enece_facpub

Part of the Controls and Control Theory Commons

How does access to this work benefit you? Let us know!

\section{Publisher's Statement}

(C) 1993 IEEE. Personal use of this material is permitted. Permission from IEEE must be obtained for all other uses, in any current or future media, including reprinting/republishing this material for advertising or promotional purposes, creating new collective works, for resale or redistribution to servers or lists, or reuse of any copyrighted component of this work in other works.

\section{Original Citation}

Gao, Z., \& Antsaklis, P. J. (February 01, 1993). Explicit asymmetric bounds for robust stability of continuous and discrete-time systems. IEEE Transactions on Automatic Control, 38, 2, 332-335.

\section{Repository Citation}

Gao, Zhiqiang and Antsaklis, P J., "Explicit Asymmetric Bounds for Robust Stability of Continuous and DiscreteTime-Systems" (1993). Electrical Engineering \& Computer Science Faculty Publications. 84.

https://engagedscholarship.csuohio.edu/enece_facpub/84

This Article is brought to you for free and open access by the Electrical Engineering \& Computer Science Department at EngagedScholarship@CSU. It has been accepted for inclusion in Electrical Engineering \& Computer Science Faculty Publications by an authorized administrator of EngagedScholarship@CSU. For more information, please contact library.es@csuohio.edu. 


\section{Explicit Asymmetric Bounds for Robust Stability of Continuous and Discrete-Time Systems}

\author{
Zhiqiang Gao and Panos J. Antsaklis
}

\begin{abstract}
The problem of robust stability in linear systems with parametric uncertainties is considered. Explicit stability bounds on uncertain parameters are derived and expressed in terms of linear inequalities for continuous systems, and inequalities with quadratic terms for discrete-times systems. Cases where system parameters are nonlinear functions of an uncertainty are also examined.
\end{abstract}

\section{INTRODUCTION}

We are interested in the stability of systems with parameter uncertainties in the state-space model and, in particular, in obtaining bounds on the uncertain parameters to guarantee the stability of the system. This problem is related to the robust stability problem of interval matrices, which has been studied by many researchers; note that [1] contains a review of this subject, including recent research results. Using these results, one can determine if a matrix with entries varying over some interval remains stable; such results however do not generally provide the range of parameters for stability, which is the problem of interest here. Most of previous results on robust stability that provide bounds on the parameter uncertainties in the state-space model to preserve stability [2]-[6] are restricted to bounds on the absolute values of the uncertain parameters; that is the corresponding stable region in the parameter space is always symmetric with respect to the origin. Clearly, this may introduce conservatism in the results, and, in fact, as it is shown later in the paper, such results can sometimes be very conservative indeed.

Progress has been made recently in obtaining less conservative parameter bounds for robust stability using the Lyapunov approach [5], [9], [10]. In particular, the bounds developed in [10] are not necessarily symmetric with respect to the origin in the parameter space, as in the previous results, and this reduces the conservatism significantly. The approaches developed in this note is based on the Lyapunov approach used in [5]. However, the stability bounds derived here are much less conservative than the one in [5], as is shown in Example 1. These bounds are different from the ones in [10] in that they are expressed explicitly in terms of the uncertain parameters, rather than a convex hull over intervals in parameter space. This is significant, since it makes it possible to further reduce the conservatism of the stability bounds in a class of problems where the knowledge of the signs and ranges of the uncertain parameters are available. It also enables us to derive a similar bound for discrete-time systems and investigate the cases where the systems parameters are nonlinear functions of an uncertainty.

Consider the state-space model for continuous-time systems with perturbation $E$

$$
\dot{x}=(A+E) x
$$

where $A$ is an $n \times n$ real Hurwitz matrix. Assume that the perturbation matrix $E$ takes the form

$$
E=\sum_{i=1}^{m} k_{i} E_{i}
$$

where $E_{i}$ are given real constant matrices; and $k_{i}$ are real uncertain parameters. The upper and lower bounds on $k_{i} i=1$, $m$ are to be found such that if $k_{i} i=1, m$ are within these bounds, the system in (1.1) remains stable; that is the eigenvalues of $(A+E)$ have negative real parts. For discrete-time systems, the state-space model has the form

$$
x(k+1)=(A+E) x(k)
$$

with $E$ defined again as in (1.2). In this case, the bounds on $k_{\text {. }}$ are to be found so that the eigenvalues of $(A+E)$ have magnitude less than one.

\section{STABILITY BOUNDS FOR CONTINUOUS AND DISCRETE-TIME SYSTEMS}

Since it is assumed that $A$ in (1.1) is Hurwitz, there exists a symmetric positive definite matrix $P$ that is the unique solution of the Lyapunov equation (see, e.g., [8])

$$
P A+A^{T} P+2 I=0
$$

Define $P_{i}$ as

$$
P_{i}=\left(E_{i}^{T} P+P E_{i}\right) / 2, \quad i=1, m \text {. }
$$

Note that $P_{i}$ are real and symmetric (Hermitian) matrices. The following theorem establishes the stability constraints on the actual uncertain parameters, $k_{i} i=1, m$. It is derived using results from the Lyapunov stability theory, via an approach similar to the one used in [5]. Let $\lambda(X)$ denote any eigenvalue of matrix $X$, and $\lambda_{\max }(X)$ and $\lambda_{\min }(X)$ the largest and smallest eigenvalues of $X$, respectively.

Theorem 1: The system in (1.1) is asymptotically stable if

$$
\sum_{i=1}^{m} k_{i} \lambda_{i} \ll 1
$$

with $\lambda_{i} i=1, m$ defined by

$$
\lambda_{i}=\left\{\begin{array}{ll}
\lambda_{\max }\left(P_{i}\right) & \text { for } k_{i} \geq 0 \\
\lambda_{\min }\left(P_{i}\right) & \text { for } k_{i}<0
\end{array} \quad i=1, m .\right.
$$


Remark: Theorem 1 gives a stability region in the parameter space, and this region is defined by the inequality in (2.3). From this inequality, it can be seen that the stability bound on one uncertain parameter is also dependent on the size of the uncertainties in other parameters. From (2.3), if there is a large uncertainty in one of the parameters, then, in general, we cannot allow large uncertainties in the rest of the uncertain parameters. The size of $\lambda_{i}$ can be viewed as a weighting factor that decides to what degree the parameter $k_{i}$ can vary. Clearly, any method that gives a single stability bound for all uncertain parameters, will introduce significant conservatism.

Before we prove Theorem 1, consider the following lemmas.

Lemma 1: Let $\alpha_{1} \leq \alpha_{2} \leq \cdots \leq \alpha_{n}, \beta_{1} \leq \beta_{2} \leq \cdots \leq \beta_{n}$, and $\gamma_{1} \leq \gamma_{2} \leq \cdots \leq \gamma_{n}$ be eigenvalues of the Hermitian matrices $A, B$, and $C=A+B$. Then

$$
\alpha_{i}+\beta_{1} \leq \gamma_{i} \leq \alpha_{i}+\beta_{n} \quad i=1, n .
$$

Proof: See [7, page 315].

Lemma 2: For any Hermitian matrices $P_{i}$, and scalars $k_{i} i=1, m$

$$
\lambda\left(\sum_{i=1}^{m} k_{i} P_{i}\right) \leq \sum_{i=1}^{m} \lambda_{\max }\left(k_{i} P_{i}\right) .
$$

Proof: This lemma can be proved by repeated applications of Lemma 1 as follows: Directly from Lemma 1, for any Hermitian matrices $A$ and $B$

$$
\lambda(A+B) \leq \lambda_{\max }(A)+\lambda_{\max }(B)
$$

and also

$$
\lambda_{\max }(A+B) \leq \lambda_{\max }(A)+\lambda_{\max }(B) .
$$

Since $P_{i}$ are Hermitian matrices, $\sum_{i=1}^{m} k_{i} P_{i}$ is a Hermitian matrix for any $m$. Thus

$$
\begin{aligned}
\lambda\left(\sum_{i=1}^{m} k_{i} P_{i}\right) & \leq \lambda_{\max }\left(k_{1} P_{1}\right)+\lambda_{\max }\left(\sum_{i=2}^{m} k_{i} P_{i}\right) \\
& \leq \lambda_{\max }\left(k_{1} P_{1}\right)+\lambda_{\max }\left(k_{2} P_{2}\right)+\lambda_{\max }\left(\sum_{i=3}^{m} k_{i} P_{i}\right) \\
& \leq \cdots \\
& \leq \sum_{i=1}^{m} \lambda_{\max }\left(k_{i} P_{i}\right) .
\end{aligned}
$$

We shall now prove Theorem 1 .

Proof of Theorem 1: Let $V(x)=x^{T} P x$, then

$$
\begin{aligned}
d V / d t & =\dot{x}^{T} P x+x^{T} P \dot{x} \\
& =[(A+E) x]^{T} P x+x^{T} P[(A+E) x] \\
& =x^{T}\left[A^{T} P+P A+E^{T} P+P E\right] x \\
& =x^{T}\left[E^{T} P+P E-2 I\right] x \\
& =x^{T}\left[\sum_{i=1}^{m} k_{i} E_{i}^{T} P+\sum_{i=1}^{m} k_{i} P E_{i}-2 I\right] x \\
& =x^{T}\left[\sum_{i=1}^{m} k_{i}\left(E_{i}^{T} P+P E_{i}\right)-2 I\right] x \\
& =2 x^{T}\left[\sum_{i=1}^{m} k_{i} P_{i}-I\right] x .
\end{aligned}
$$

Let

$$
M:=\left[\sum_{i=1}^{m} k_{i} P_{i}-I\right] .
$$

Note that $M$ is an $n \times n$ real and symmetric matrix. For the system in (1.1) to be asymptotically stable, $d V / d t$ must be negative, or, equivalently, $M$ must be negative definite. Since a symmetric matrix is negative definite if, and only if, all its eigenvalues are negative, we need

$$
\lambda\left(\sum_{i=1}^{m} k_{i} P_{i}-I\right)<0
$$

which is true if, and only if

$$
\lambda\left(\sum_{i=1}^{m} k_{i} P_{i}\right)<1
$$

that is every eigenvalue of $\sum_{i=1}^{m}, k_{i} P_{i}$ must be less than 1. In view of Lemma 2

$$
\lambda\left(\sum_{i=1}^{m} k_{i} P_{i}\right) \leq \sum_{i=1}^{m} \lambda_{\max }\left(k_{i} P_{i}\right)
$$

and since

$$
\begin{gathered}
\lambda_{\max }\left(k_{i} P_{i}\right)= \begin{cases}k_{i} \lambda_{\max }\left(P_{i}\right) & \text { for } k_{i} \geq 0 \\
k_{i} \lambda_{\min }\left(P_{i}\right) & \text { for } k_{i} \leq 0\end{cases} \\
=k_{i} \lambda_{i} \\
\sum_{i=1}^{m} \lambda_{\max }\left(k_{i} P_{i}\right) \leq \sum_{i=1}^{m} k_{i} \lambda_{i} .
\end{gathered}
$$

Hence, the system in (1.1) is stable if

$$
\sum_{i=1}^{m} k_{i} \lambda_{i}<1
$$

The significance of this theorem is that it takes into consideration the directional information which is often available in practice, thus reducing the conservatism found in earlierliterature results. To demonstrate this, it is shown below that the stability bound obtained here is always less than or equal to one of the bounds proposed in [5], namely

$$
\sum_{i=1}^{m}\left|k_{i}\right| \sigma_{\max }\left(P_{i}\right)<1
$$

where $\sigma_{\max }(\cdot)$ denotes the largest singular value; see also Example 1.

Since $P_{i}$ is a Hermitian matrix, $\sigma_{\max }\left(P_{i}\right)=\max \{|\lambda|, \lambda \in$ $\left.\lambda\left(P_{i}\right)\right\}$. Hence, for $\lambda_{i}$ defined in (2.2), we have $\left|\lambda_{i}\right| \leq \sigma_{\max }\left(P_{i}\right)$. Therefore

$$
\sum_{i=1}^{m} k_{i} \lambda_{i} \leq \sum_{i=1}^{m}\left|k_{i}\right|\left|\lambda_{i}\right| \leq \sum_{i=1}^{m}\left|k_{i}\right| \sigma_{\max }\left(P_{i}\right) .
$$

In other words, if (2.12) is satisfied, then (2.3) is satisfied. That is, the stability bound found in Theorem 1 is always less conservative than the one in (2.12). Clearly, the reason the new stability bound is less conservative is that it takes the directional information into consideration. This can be explained by the fact that as a parameter varies in different directions, it affects the system stability differently. This can be easily shown using, for example, the root-locus technique, where it is well known that, for differ- 
ent signs of the parameter, the root locus is completely different; that is, the effect of a single parameter $k$ in $A$ on its eigenvalues can be completely different for the same $|k|$ and opposite sign. Any tests, therefore, that ignore the sign are bound to be conservative in typical cases.

Moreover, from the stability conditions (2.3), if for some $k_{j}$ we have

$$
k_{j} \lambda_{j} \leq 0
$$

then such uncertain parameters will not affect the system stability. This is because for $k_{j}$ that satisfy $(2.13)$

$$
\sum_{i=1}^{m} k_{i} \lambda_{i} \leq \sum_{i=1, i \neq j}^{m} k_{i} \lambda_{i}
$$

and, therefore, the stability criteria in (2.3) becomes

$$
\sum_{i=1, i \neq j}^{m} k_{i} \lambda_{i}<1
$$

In (2.14) the conservatism is further reduced since there are fewer parameters to be considered. Furthermore, if the lower bounds $a_{j}(\geq 0)$ of the absolute values of such $k_{j}$

$$
\left|k_{j}\right| \geq a_{j}, \quad \text { for some } j
$$

are known, then the uncertainties in $k_{j}$ can actually be used in offsetting the destabilizing effect of other uncertain parameters. This is formalized in the corollary below.

Corollan: Assume there exist $k_{j}$ that satisfy (2.13) and (2.15) for some $j$. Then the system in (1.1) is stable if

$$
\sum_{i=1, i \neq j}^{m} k_{i} \lambda_{i}<1+\sum_{j} a_{j}\left|\lambda_{j}\right|
$$

Proof: The stability condition $\sum_{i=1}^{m}, k_{i} \lambda_{i}<1$ is equivalent to

$$
\sum_{i=1, i \neq j}^{m} k_{i} \lambda_{i}<1-\sum_{j} k_{j} \lambda_{j}
$$

and for $k_{j}$ that fulfill $k_{j} \lambda_{j} \leq 0$ and $\left|k_{j}\right| \geq a_{j}$, we have

$$
\left(-\sum_{j} k_{j} \lambda_{j}\right)=\sum_{j}\left|k_{j}\right|\left|\lambda_{j}\right| \geq \sum_{j} a_{j}\left|\lambda_{j}\right| .
$$

Hence, $\sum_{i=1}^{m} k_{i} \lambda_{i}<1$ is true, and system (1.1) is stable, if $\sum_{i=1, i \neq j}^{m} k_{i} \lambda_{i}<1+\sum_{j} a_{j}\left|\lambda_{j}\right|$.

In practical control problems, system parameters are typically associated with physical entities. It is reasonable to assume that signs and ranges of many parameters are known although their values are uncertain. From (2.13)-(2.16), it is shown how this information can be utilized in achieving less conservative bounds. This is only possible because the uncertain parameters $k_{i}$ are explicitly expressed in the inequality (2.3), and it cannot be accomplished using existing results such as in [10].

Example 1: Let $m=2$, with $A, E_{1}$, and $E_{2}$ given as

$$
A=\left[\begin{array}{cc}
-3 & -2 \\
1 & 0
\end{array}\right], \quad E_{1}=\left[\begin{array}{cc}
-1 & -1 \\
0 & 0
\end{array}\right], \quad E_{2}=\left[\begin{array}{ll}
1 & 1 \\
0 & 0
\end{array}\right] \text {. }
$$

The eigenvalues of $p_{i}$ defined in (2.2) are

$$
\lambda\left(P_{1}\right)=\{-1,0\}, \quad \text { and } \lambda\left(P_{2}\right)=\{1,0\}
$$

and, therefore, the stability bounds given by (2.14) are

$$
\begin{array}{ll}
k_{2}<1 & \text { for } k_{1}>0, k_{2}>0 \\
\forall k_{1}, k_{2} & \text { for } k_{1}>0, k_{2}<0 \\
k_{2}-k_{1}<1 & \text { for } k_{1}<0, k_{2}>0 \\
k_{1}>-1 & \text { for } k_{1}<0, k_{2}<0
\end{array}
$$

The corresponding stability bound obtained in [5] is

$$
\left|k_{1}\right|+\left|k_{2}\right|<1 \quad \text { for any } k_{1}, k_{2}
$$

and the actual stability bound in this case is $k_{2}-k_{1}<2$. Note that the stability region obtained using the new method is open to infinity, See Fig. 1.

Example 2: Let $m=2$, with $A, E_{1}$, and $E_{2}$ given as

$A=\left[\begin{array}{cc}-3 & -2 \\ 1 & 0\end{array}\right], \quad E_{1}=\left[\begin{array}{cc}-5 & 1 \\ 1 & -1\end{array}\right]$

$$
E_{2}=\left[\begin{array}{cc}
7.5 & -1.5 \\
-1.5 & 1.5
\end{array}\right]
$$

Also, assume that $k_{1}$ has a lower bound, $k_{1} \geq 2$. The eigenvalues of $P_{i}$ are

$$
\lambda\left(P_{1}\right)=(-2,-2), \quad \text { and } \lambda\left(P_{2}\right)=\{3,3\}
$$

therefore, from (2.4), $\lambda_{1}=-2$ and $\lambda_{2}=3$. Note that since $\lambda_{1} k_{1}<0, k_{1}$ will not affect the system stability. By Corollary 1 , the stability bound is

$$
k_{2} \lambda_{2}<1+2 * 2=5
$$

or

$$
k_{2}<5 / 3
$$

This example shows that not only do some uncertainties not destabilize the system, but they also play a role of offsetting the destabilizing effect of other uncertainties. Here, the presence of the uncertainty $k_{1}$ actually increases the stability bound of $k_{2}$ from $k_{2}<1 / 3$ to $k_{2}<5 / 3$, where $k_{2}<1 / 3$ is the stability bound obtained without taking $k_{1}$ into consideration. Note that such an increase could not be obtained using the bounds derived in [10].

The above results were derived for continuous-time linear systems. A similar approach can be used for discrete-time linear systems with parametric uncertainties in the state-space model (1.3). This is briefly discussed below, and corresponding results for the discrete-time case are outlined.

Define the Lyapunov function as $V(x)=x^{T} P x$, where $P$ is the solution of the Lyapunov equation for the discrete-time system (see, e.g., [8]).

$$
A^{T} P A-P+2 I=0 .
$$

Then, it can be shown that

$$
\begin{aligned}
\Delta V & =V(x(k+1))-V(x(k)) \\
& =2 x^{T}\left[\sum_{i} k_{i} P_{i}+\sum_{i, j} k_{i} k_{j} F_{i j}-I\right] x
\end{aligned}
$$

where $P_{i}$ is defined as

$$
P_{i}:=\left(E_{i}^{T} P A+A^{T} P E_{i}\right) / 2, \quad i=1, \cdots, m
$$

and

$$
F_{i j}:=E_{i}^{T} P E_{j} / 2 .
$$

Note that $\Delta V$ in (2.18) has a similar form as its counterpart $d V / d t$ in the case of continuous-time systems, and a similar approach can be used here to derive the stability bounds. The following result, which is applicable to the discrete-time system (1.3), is the counterpart of Theorem 1 , and can be proved in a similar way. 


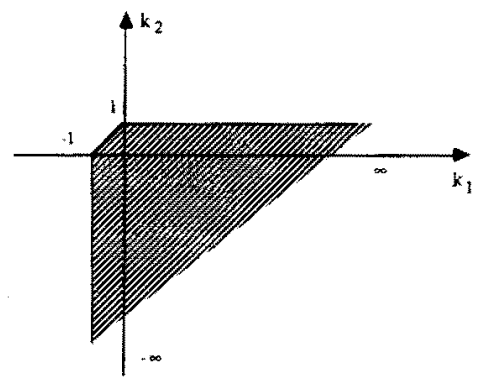

Fig. 1. Example 1, stability bounds of $k_{1}$ and $k_{2}$.

Theorem 2: The system in (1.3) is asymptotically stable if

$$
\sum_{i} k_{i} \lambda_{i}+\sum_{i, j} k_{i} k_{j} f_{i j}<1
$$

with $\lambda_{i}$ defined in (2.4), $P_{i}$ defined in (2.19), and $f_{i j}$ defined as

$$
f_{i j}=\left\{\begin{array}{ll}
\lambda_{\max }\left(F_{i j}\right) & \text { for } k_{i} k_{j} \geq 0 \\
\lambda_{\min }\left(F_{i j}\right) & \text { for } k_{i} k_{j}<0
\end{array} \quad i, j=1, m .\right.
$$

Example 3: Let the nominal discrete-time system be

$$
x(k+1)=\left[\begin{array}{cc}
0.5 & 0 \\
0 & -0.5
\end{array}\right] x(k)
$$

and the perturbation be

$$
E=k_{1}\left[\begin{array}{cc}
-1 & 0 \\
0 & 1
\end{array}\right]+k_{2}\left[\begin{array}{cc}
1 & 0 \\
0 & -1
\end{array}\right]
$$

Then, from (2.19)-(2.22), we have $P=\operatorname{diag}(8 / 3,8 / 3)$, $P_{1}=\operatorname{diag}(-4 / 3,-4 / 3), P_{2}=-P_{1}, F_{11}=F_{22}=P_{2}, F_{12}=F_{21}$ $=P_{1}, \lambda_{1}=-4 / 3, \lambda_{2}=4 / 3, f_{11}=f_{22}=4 / 3$, and $f_{12}=f_{21}=$ $-4 / 3$. Applying (2.21), the stability constraint on $k_{1}$ and $k_{2}$ is

$$
\left(k_{2}-k_{1}\right)^{2}+\left(k_{2}-k_{1}\right)<3 / 4
$$

or equivalently

$$
\begin{gathered}
k_{2}-k_{1}<1 / 2 \\
k_{2}-k_{1}>-3 / 2
\end{gathered}
$$

Interestingly, this stability region, derived by applying the new stability bound, is exactly the same as the actual one; of course in other examples this may not be the case.

\section{SYSTEMS WITH NONLINEARLY DEPENDENT UNCERTAIN PARAMETERS}

It is shown in the following how the above results can be used to solve more complicated problems in robust stability of dynamic systems. Consider the following problem: given the uncertain system

and

$$
\dot{x}=(A+E(r)) x
$$

$$
E(r)=\sum_{i=1}^{m} k_{i}(r) E_{i}
$$

where $A \subseteq R^{n \times n}$ is Hurwitz; $k_{i}(r) i=1, m$ are given continuous functions of $r \in \boldsymbol{R}$; and $E_{i} \in \boldsymbol{R}^{n \times n} i=1, m$ are given constant matrices, determine the stability region $\Psi \subset \boldsymbol{R}$ such that for $r \in \Psi$, (3.1) remains stable.

Note that here the uncertain parameters are functions of one parameter $r$. A similar approach can be taken when they depend on more than one parameters, however, this will not be discussed in this note. It can be easily shown that for the more complicated perturbation matrix $E(r)$ in (3.2), Theorem 1 still holds, and the corresponding stability constraints are, in this case

$$
\sum_{i=1}^{m} k_{i}(r) \lambda_{i}<1
$$

Inequality (3.3) serves as a starting point in the stability analysis of systems (3.1) and (3.2). It is significant because it enables us to study the effect of $r$ on the system stability. Such problems cannot be solved directly by using existing methods, since the uncertain parameters $k_{i}(r)$ are, in general, nonlinearly dependent to each other via $r$.

There are two possible methods to obtain the stability region $\Psi$. One is an analytical method, by which the bounds for $r$ are explicitly derived from (3,3). However, this is not always possible due to the arbitrariness of the functions $E(r)$ and $k_{i}(r)$. The other method is a graphical approach, where, with the help of computer software packages such as Matlab, we can easily plot $f(r)=\sum_{i=1}^{m} k_{i}(r) \lambda_{i}$ as a function of $r$ and, therefore, determine the stability region $Y$, which is the region that satisfies $f(r)<1$.

Example 4: Consider the stability of the system

$$
\dot{x}=\left(\left[\begin{array}{cc}
-3 & -2 \\
1 & 0
\end{array}\right]+k_{1}(r)\left[\begin{array}{cc}
-1 & -1 \\
0 & 0
\end{array}\right]+k_{2}(r)\left[\begin{array}{ll}
1 & 1 \\
0 & 0
\end{array}\right]\right) x
$$

where the system matrix is affected by the uncertainty $r$ through the nonlinear functions $k_{1}(r)=e^{r}$ and $k_{2}(r)=r^{3}$. By Theorem 1 , we first calculate the eigenvalues of $P_{i}$ defined in (2.1)

$$
\lambda\left(P_{1}\right)=\{-1,0\}, \quad \text { and } \lambda\left(P_{2}\right)=\{1,0\}
$$

and then the stability bounds given by (3.3) can be found as

$$
\begin{array}{ll}
k_{2}(r)<1, & \text { for } k_{1}(r)>0, k_{2}(r)>0 \\
\forall k_{1}(r), k_{2}(r), & \text { for } k_{1}(r)>0, k_{2}(r)<0 \\
k_{2}(r)-k_{1}(r)<1, & \text { for } k_{1}(r)<0, k_{2}(r)>0 \\
k_{1}(r)>-1, & \text { for } k_{1}(r)<0, k_{2}(r)<0 .
\end{array}
$$

Substituting $k_{1}(r)=e^{r}$ and $k_{2}(r)=r^{3}$ in the above inequalities, the equivalent stability constraints in terms of $r$ are

$$
r^{3}<1, \quad \text { for } e^{r}>0, r^{3}>0
$$

and, by simple manipulation, the stable region for the uncertainty $r$ is found to be $-\infty<r<1$, which is rather close to the exact stability bound $-\infty<r<1.25$.

\section{REFERENCES}

[1] M. Mansour "Robust stability of interval matrices," in Proc. 28th IEEE Conf. Decision Contr., Dec. 1989, pp. 46-51.

[2] J. M. Martin, "State-space measures for stability robustness," IEEE Trans. Automat. Contr., vol. AC-32, pp. 509-512, June 1987.

[3] L. Qiu and E. J. Davison, "New perturbation bounds for the robust stability of linear state-space models," in Proc. 25th IEEE Conf. Decision Contr., Dec. 1986, pp. 751-755.

[4] R. K. Yedavalli, "Stability rpbistmess measures under dependent uncertainty," in Proc. Amer. Contr. Conf., June 1988, pp. 820-823.

[5] K. Zhou and P. P. Khargonekar, "Stability robustness bounds for linear state-space models with structured uncertainty," IEEE Trans. Automat. Contr, vol. AC-32, pp. 621-623, July 1987.

[6] P. Bauer and K. Premaratne, "Robust stability of time-variant interval matrices," in Proc. 29th IEEE Conf. Decision Contr., Dec. 1990 , pp. 334-335.

[7] G. W. Stewart, Introduction to Matrix Computations. New York: Academic, 1973.

[8] S. Barnett and C. Storey, Matrix Methods in Stability Theory. New York: Barnes \& Noble, 1970.

[9] Z. Gao, "Reconfigurable control systems: analysis and design," Ph.D. dissertation, Dept of Electric. Eng., Univ. Notre Dame, IN, Aug. 1990.

[10] D. S. Bernstein and W. M. Haddad, "Robust stability and performance analysis for linear dynamic systems," IEEE Trans. Automat. Contr., vol. 34, pp. 751-758, July 1989. 